\section{Spargel als Allergieauslöser}

Spanien ist mit über 61.000 Tonnen pro Jahr der viertgrößte Spargelproduzent der Welt. Daher verwundert es nicht, dass dort Kontaktallergien gegen das Gemüse auftreten. Betroffen sind vor allem Arbeiter, die in der Konservenherstellung beschäftigt sind.

A llergologen aus Pamplona dokumentierten 27 Patienten mit allergischen Reaktionen gegen Spargel. Sie bestimmten dazu in allen Fällen spezifische IgE-Antikörper im Serum und führten Prick- und Epikutantests durch. Dabei kamen sowohl ein Gesamt-Spargelextrakt als auch die Lipidtransferproteine Aspa o 1.01 und Aspa o 1.02 zum Einsatz, die als wichtige Allergene des Liliengewächses gelten.

Von den 27 Patienten litten acht unter einer Kontaktdermatitis, 17 hatten eine IgE-vermittelte Allergie und zwei sowohl eine Kontaktdermatitis als auch eine Typ-I-Allergie. Die Patienten mit Kontaktdermatitis reagierten im Epikutantest positiv auf den Spargelextrakt, nicht aber auf die Proteinfraktion.
Von den 19 Patienten mit Typ-IAllergie zeigten zehn eine Kontakturtikaria im Epikutantest und zehn litten unter respiratorischen Symptomen. Durch die inhalative Provokation mit Spargel konnten davon acht als Asthmatiker klassifiziert werden, zwei litten ausschließlich unter Rhinitis.

Vier Patienten zeigten eine allergische Reaktion unmittelbar nach dem Verzehr von Spargel. Diese äußerte sich in Form einer Anaphylaxie oder als orale Kontakturtikaria.

Von den Patienten mit einem positiven Pricktest gegen den Gesamt-Spargelextrakt reagierte nur rund die Hälfte auch auf die Lipidtransferproteine. Immunoblot-Untersuchungen legten nahe, dass auch Glykoproteine im Bereich

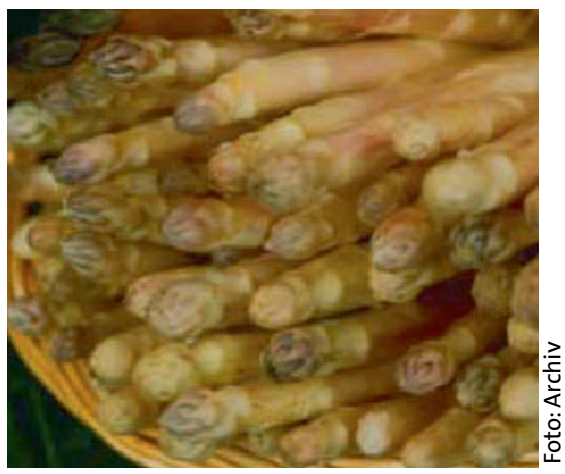

„Essbares Elfenbein“ - das Liliengewächs Spargel hat viele Liebhaber unter den Gourmets.

von $45-70 \mathrm{kDa}$ als Allergene eine Rolle spielten.

Fazit: Spargel kann Allergie in Form von Kontaktdermatititiden oder IgEvermittelten Reaktionen hervorrufen. Betroffen waren hier vor allem beruflich exponierte Personen. mha

Tabar Al et al. Diversity of asparagus allergy: clinical and immunological features. Clin Exp Allergy 2004; 34: 131-6

\section{Erst falsch gegessen - dann falsch versorgt}

Anaphylaktoide Reaktion ist die Diagnose bei bis zu 2\% aller Patienten in Notfallambulanzen. Eine retrospektive Kohortenstudie untersuchte jetzt die Behandlung von Anaphylaxie aufgrund einer Nahrungsmittelallergie in Nordamerika.

Fisol orscher sichteten die Akten von 21 US-amerikanischen und kanadischen Notfallambulanzen und selektierten aus den dort dokumentierten Daten von 5.296 Patienten mit einer Nahrungsmittel-induzierten anaphylaktischen Reaktion randomisiert 678 Fälle, um sie einer standardisierten Analyse zu unterziehen. Bei über $90 \%$ der Patienten konnte das verantwortliche Allergen identifiziert werden: Am häufigsten waren Nüsse (21\%) die Auslöser, gefolgt von Krustazeen (19\%), Früchten/Gemüse (12\%) und Fisch (10\%).

Obwohl eine anaphylaktoide Reaktion potenziell lebensbedrohlich ist, wurden nur 18\% der Patienten mit dem Notarztwagen eingeliefert. Die mediane Zeit bis zum Aufsuchen der Ambulanz betrug 2 Stunden. Die Behandlung erfolgte bei $72 \%$ der Fälle mit einem Antihistaminikum, bei $48 \%$ durch systemische Glukokortikoide und bei 16\% mit Adrenalin - subkutan oder intramuskulär. 33\% der Patienten erhielten eine bronchodilatierende $\mathrm{Me}$ dikation mit Beta-Agonisten oder Anticholinergika. Von den Patienten mit einer schweren anaphylaktischen Reaktion - 55\% der Kohorte - wurden 24\% mit Adrenalin behandelt. Ein Todesfall trat nicht auf.

97\% der Patienten wurden nach median 145 Minuten wieder nach
Hause geschickt. Immerhin 40\% der Patienten erhielten zuvor den Rat, das Allergen in Zukunft zu meiden, 16\% bekamen ein Rezept über Adrenalin zur Selbstinjektion und 12\% wurden zum Allergologen überwiesen. Nur insgesamt 12 Patienten $(2 \%)$ kamen in den Genuss aller drei Präventionsempfehlungen, die immerhin als Leitlinien für derartige Notfälle vorliegen.

Fazit: Die Versorgung Nahrungsmittel-induzierter anaphylaktoider Reaktionen erfolgte nur in geringem Maße leitlinienkonform, selbst bei den schweren Formen wurde lediglich bei einem Viertel der Patienten Adrenalin angewandt. Aufklärungsbedarf ist offensichtlich gegeben - und die in Nordamerika bestehende Empfehlung genereller Adrenalininjektion bei Anaphylaxie sollte überdacht werden. $\quad b k$

Clark S et al. Multicenter study of emergency department visits for food allergies. J Allergy Clin Immunol 2004; 113: 347-52 